\title{
Impacto ambiental por el incremento demográfico en el municipio de San Gil, Santander
}

\section{Environmental impact on the population increase in the municipality of San Gil, Santander}

\author{
Yuleimy Ramírez Ordoñez ${ }^{1}$
}

\begin{abstract}
Resumen
Ésta investigación busca determinar el impacto ambiental ocasionado por el incremento demográfico en el municipio de San Gil, Santander durante los últimos diez años, mediante la identificación y descripción de las variables ambientales afectadas por el crecimiento demográfico, la evaluación de impactos ambientales a través de la matriz de Leopold. Finalmente se formularon lineamientos de gestión para orientar el desarrollo municipal.
\end{abstract}

El Municipio de San Gil, catalogado como generador de diversos servicios especialmente el de turismo, alberga el 56\% de la población de la provincia de Guanentá (Plan Prospectivo Guanentá 2025, 2010), con 45.956 habitantes; dada su influencia como capital de provincia y principal eje comercial de la región. La dinámica poblacional que se desarrolla en el municipio mantiene la misma tendencia nacional: la mayor población se encuentra acentuada en la zona urbana, alcanzando el $84 \%$ (Planeación Municipal, 2012).

El aumento de la población trae consigo la necesidad de mayor requerimiento de alimentos, servicios públicos, fuentes de trabajo, educación, servicios de salud, aprovisionamiento de equipamiento e infraestructura, entre otros, y como consecuencia el aumento de vertimientos líquidos, de Residuos Sólidos Urbanos (RSU), emisiones de gases de efecto invernadero (GEI), pérdida de cobertura vegetal y desaparición de especies, y en general el desmejoramiento de la calidad de vida. En San Gil se consume agua en un promedio de 156 1/hab/día, se vierten aguas residuales en $95,75 \mathrm{l} / \mathrm{s}^{-1}$ al río Fonce, principal fuente receptora sin tratamiento previo, se generan aproximadamente 12.721 ton/año $0^{-1}$ de RSU; su degradación produce en promedio 772,419 ton/año ${ }^{-1}$ de GEI y por consumo energético eléctrico se emiten $6.123,891$ ton $/ \mathrm{año}^{-1} \mathrm{de}$ GEI.

Palabras clave: Gestión ambiental; residuos sólidos; crecimiento demográfico; contaminación ambiental; variables ambientales.

\begin{abstract}
This research seeks to determine the environmental impact caused by population increase in the municipality in San Gil, Santander (Colombia) during the last ten years, through the identification and description of environmental variables affected by population growth, environmental impact assessment through Leopold matrix. Finally management guidelines were formulated to guide municipal development.

The Municipality of San Gil, listed as a generator of various services especially tourism, home to $56 \%$ of the population of the province of Guanentá (Guanentá Perspective Plan 2025, 2010), with 45,956 inhabitants; given its influence as a provincial capital and main commercial hub of the region. The population dynamics taking place in the town maintains the same national trend: the population is pronounced in urban areas, reaching $84 \%$ (Municipal Planning, 2012).
\end{abstract}

1 - Ingeniera Ambiental, Fundación Universitaria de San Gil Unisangil Km2 Vía San Gil Charalá, yramirez@unisangil.edu.co 
The increase in population brings with it the need for increased requirement for food, utilities, jobs, education, health services, supply of equipment and infrastructure, among others, and as a result increasing liquid discharges, Solid Waste (RSU), emissions of greenhouse gases (GHGs), loss of plant cover and species loss, and general deterioration in the quality of life. In San Gil water consumed in an average of 1561 / capita / day wastewater is discharged at $95.751 / \mathrm{s}^{-1}$ to Fonce River, the main source without receiving treatment, generate approximately 12,721 tons / year ${ }^{-1} \mathrm{MSW}$; degradation occurs on average 772.419 tonnes / year ${ }^{-1} \mathrm{GHG}$ and electric energy consumption 6123.891 tonnes $/$ year $^{-1}$ GHG emitted.

Keywords: Environmental management; solid waste; population growth; environmental pollution; environmental variables.

\section{Introducción}

En la escala geológica del tiempo, la presencia del hombre sobre la tierra ha sido un episodio esporádico, sin embargo, su paso por el planeta, sobre todo en las últimas décadas, ha tenido consecuencias como los impactos de meteoritos y cometas, los cambios de polaridad y del eje de la tierra, la explotación de supernovas cercanas y las glaciaciones, que son consideradas como algunas de las probables causas de las extinciones masivas que ocurrieron en tiempos remotos (Arias, 2010).

Como es usual, la pérdida de biodiversidad se debe a la falta de organización del territorio que permita la identificación de ecosistemas estratégicos para el desarrollo de la misma vida humana y la reorganización del espacio físico y natural asegurando que sea sostenible y satisfaga las necesidades del presente sin comprometer la capacidad de las futuras generaciones para satisfacer las propias (Bennett \& Michael, 1999).

El crecimiento demográfico se considera el principal responsable de los daños que sufre la naturaleza. (Bloom, 2006). El impacto de la población sobre el medio ambiente se relaciona con variables tales como: el consumo de recursos, la producción de RSU y de contaminantes en cualquiera de sus estados. Causa un aumento de la utilización del medio ambiente (Georg, 2002). Sin duda, cuantas más personas haya, será mayor la producción de residuos sólidos y líquidos, lo que implica cargas adicionales para la capacidad receptora de los ecosistemas (Weltbank, 1992).

Todas las actividades humanas provocan emisiones de GEI, principalmente, las asociadas al suministro de energía, silvicultura, industria, agricultura y transporte. Las emisiones de $\mathrm{CO}_{2}$ y los residuos sólidos en las ciudades son indicadores de una utilización masiva del medio ambiente que al parecer crece con el aumento del ingreso económico. Esto se debe a que todavía no existen suficientes incentivos para lograr un cambio en el comportamiento (Weltbank, 1992).

Debido a la actividad humana, actualmente se están perdiendo hasta mil veces más especies de lo que ocurriría si solo actuaran causas naturales. Uno de cada cuatro mamíferos y uno de cada tres anfibios se encuentra en amenaza de extinción. Cuando las especies se extinguen, ni siquiera el inmenso poder del dinero y de la ciencia moderna pueden reversarlo (Ponce, 2010).

El crecimiento demográfico y los problemas ambientales, son un reto para las instituciones locales responsables del desarrollo social integral. El propósito de esta investigación fue determinar el impacto ambiental asociado al incremento demográfico en el municipio de San Gil en la última década, mediante la identificación y evaluación de variables ambientales, así mismo proponer lineamientos de gestión acordes para el desarrollo municipal.

\section{Materiales y Métodos}

El municipio de San Gil ubicado al sur oriente del departamento de Santander a $6^{\circ} 33^{\prime}$ de latitud norte y $73^{\circ} 8^{\prime}$ de longitud occidental, con temperatura promedio de 24 grados centígrados, capital de la provincia de Guanentá y principal eje comercial y de servicios en la región.

Éste estudio se enmarca dentro del tema de investigación dirigida de tipo descriptiva, encaminada al área de gestión ambiental. Es una investigación teórica cuanti-cualitativa enfocada a identificar el impacto ambiental que provoca el incremento de la población en el municipio de San Gil, Santander en los últimos diez años, a través de la identificación de variables ambientales afectadas y su posterior evaluación. 
Para el desarrollo del estudio se asumió una tasa de crecimiento demográfico igual a $1,5 \%$ de acuerdo a proyecciones realizadas por el Departamento Administrativo Nacional de Estadísticas (DANE) en el año 2005. Para la identificación de las variables ambientales impactadas por el incremento demográfico en San Gil se analizaron los renglones económicos que mantiene activa la población del municipio, tanto del sector urbano y rural, se consultaron bases de datos y registro de consumo de servicios públicos de la última década, revisión de permisos de aprovechamiento forestal y concesiones de agua, revisión de planes de ordenamiento, planes de gestión integral de residuos sólidos, plan de saneamiento y manejo de vertimientos, planes de acción y prospectiva de la provincia, análisis de proyecciones de población residente y flotante, entre otros documentos que se obtuvieron de la revisión bibliométrica y realización de entrevista a instituciones como la Electrificadora de Santander, ESSA, Empresa de acueducto, alcantarillado y aseo, ACUASAN, Corporación autónoma regional de Santander, CAS, alcaldía municipal de San Gil, fundación universitaria de San Gil, UNISANGIL.

Para el análisis y presentación de los datos se utilizaron tablas y figuras de la herramienta excel, permitiendo mejor interpretación de la información hallada, la cual fue tomada como valores promedio. Usando la matriz de Leopold se realizó el análisis de las variables agua, suelo, aire, flora, fauna y energía eléctrica, susceptibles de alterarse respecto de las actividades de la población sangileña. La valoración de las variables se realizó de -5 a 5 . (donde -5 es un alto impacto negativo, 0 indica que no hay impacto y 5 es un alto impacto negativo)

Utilizando los factores del Panel Intergubernamental de Cambio Climático (IPCC) - Unidad de Planeación Minero Energética (UPME) y Organización Latinoamericana de Energía (OLADE), se calculó las emisiones de GEI por disposición final de RSU y consumo energético eléctrico.

Finalmente se formularon lineamientos de gestión para orientar el desarrollo municipal.

\section{Resultados y Discusión}

San Gil ha sido el municipio de mayor influencia en la región, como capital turística y capital de la provincia de Guanentá ha mostrado un auge importante y que contribuye al desarrollo económico del departamento de Santander.
El Municipio de San Gil pasó de tener 41.045 a 45.956 habitantes en la última década de acuerdo a las proyecciones del DANE (2005), sin embargo en épocas festivas y decembrinas se suman entre 15.000 y 30.000 habitantes, correspondiente a población flotante producto de la influencia del turismo (Oficina de cultura y turismo, 2012).

Con el incremento de la población aumentó el uso del suelo rural; el 45,3\% del suelo en el municipio es sobreexplotado, el $6 \%$ es subutilizado y el $48,53 \%$ presenta uso adecuado (PBOT, 2003), más del 70\% es predominantemente agrario. El café es el producto más importante del municipio con una cobertura del $80,6 \%$ de las veredas; genera el $60 \%$ del empleo rural y el $80 \%$ de los caficultores son pequeños productores de menos de 5 hectáreas, sin embargo se presentan cultivos semestrales como el fríjol, el maíz y tabaco que ocupan el $19,4 \%$ del suelo rural (secretaría de planeación municipal, 2012).

La sobreutilización y explotación convencional del suelo, el uso de fertilizantes y agentes químicos empleados para lograr los cultivos, provocan erosión del terreno y definitivamente bajo rendimiento en nuevas fases productivas, presumiendo la necesidad de introducir técnicas sostenibles que promueva la producción agropecuaria en el municipio.

Las técnicas de cultivo utilizadas actualmente incluyen, quemas para preparación de terreno, expansión de la frontera agrícola en busca de mejores suelos y conflictos por servicios de riego, debido a la desaparición de las principales fuentes hídricas e intensidad de la época de estiaje. La mayoría de los predios rurales no cuentan con estrategia de almacenamiento de agua para uso agrícola.

La actividad minera, especialmente la relacionada con el aprovechamiento de arcillas de manera artesanal e ilegal, es uno de los principales portantes de sólidos sedimentables al río Fonce, principal fuente hídrica de la región. Existen aproximadamente 300 familias que se dedican a ésta actividad, siendo su principal fuente económica.

La zona urbana del municipio abarca el 5\% del territorio y debido al crecimiento urbanístico en la última década se generó un déficit de espacio público de 66.8\% (Plan de Desarrollo 2012-2015). Como es usual cuando se presenta migración hacia los cascos urbanos hay necesidad de satisfacer la disponibilidad de vivienda de la población migrante, lo que provoca el asentamiento en zonas prohibidas y de alto riesgo. 
Se observó la construcción de viviendas localizadas en laderas del rio Fonce en áreas que corresponden con la ronda de protección de 30 metros.

En San Gil, el sistema de acueducto municipal en los últimos 10 años pasó de tener 10.149 suscriptores a 13.710 , lo que demuestra un aumento en la demanda del servicio en no menos del $30 \%$. El sistema de acueducto, para suplir las necesidades de la población urbana y tan sólo el $5 \%$ de la población rural toma el agua de 2 fuentes principalmente: la quebrada Curití, la cual en época de estiaje no reduce el caudal y la quebrada Cuchicute la cual reduce el caudal hasta un $70 \%$ afectando los requerimientos del recurso y otorgados en concesión, sin embargo como es compromiso atender toda la demanda, la empresa de acueducto toma la cantidad faltante del río Fonce para poder suplir la necesidad. En la figura 1 se presenta el aumento del consumo facturado en el municipio en los últimos años.

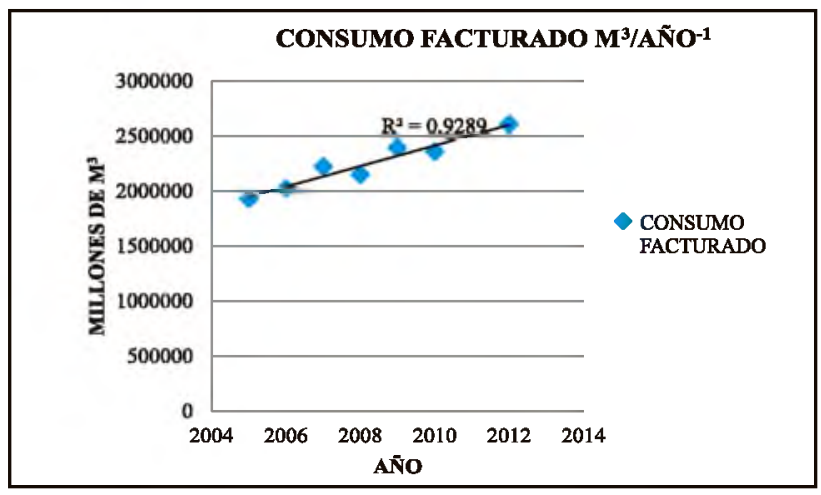

Figura 1. Consumo factura $\mathrm{m} 3$ /año

El sistema de acueducto presenta deficiencias en la red de conducción, el 50\% del caudal captado se pierde por fugas y aún existen redes con una antigüedad hasta de 30 años, no obstante la empresa de acueducto, aseo y alcantarillado del municipio facturó para el año 2012 en promedio 2.608.352 $\mathrm{m}^{3}$ /año equivalente a $156 \mathrm{l} / \mathrm{hab} /$ día, siendo el estrato 3 el mayor consumidor

Se señala que, el 95\% de la población rural no cuenta con un sistema de potabilización, lo que demuestra la ausencia de un desarrollo planificado que integre todos los actores involucrados del servicio, sin importar el comportamiento de los mismos. En el municipio se desconoce el potencial de bienes y servicios ambientales y de igual forma las demandas que pueden alcanzar a suplir con el incremento demográfico.

Por otra parte, no se ha implementado un sistema de tratamiento para el agua residual que proviene de los hogares, instituciones públicas y privadas y de la industria. De acuerdo al plan de saneamiento y manejo de vertimientos del municipio de San Gil, en promedio se vierten $95,73 \mathrm{l} / \mathrm{s}$ que corresponden a 27 vertimientos, los cuales son recogidos por un sistema de colectores y luego descargados en uno solo directamente al río Fonce. La composición típica de los vertimientos es de $2.662,4 \mathrm{mg} / \mathrm{L}$ de sólidos suspendidos totales y $5.885,6 \mathrm{mg} / \mathrm{L}$ de DBO5, las cargas están en $722,6 \mathrm{~kg} /$ día de sólidos suspendidos totales y $1.483,8 \mathrm{~kg} /$ día de $\mathrm{DBO}_{5}$ (PSMV, 2009).

El incremento de la población en el municipio de San Gil en la última década provocó el aumento de la generación de RSU, en 2006 se generaron 9.555,83 tonelada mientras que para el año 2012 se registraron $12.721,49$ toneladas lo que significa un aumento del $33 \%$ en el período evaluado como se observa en la figura 2.

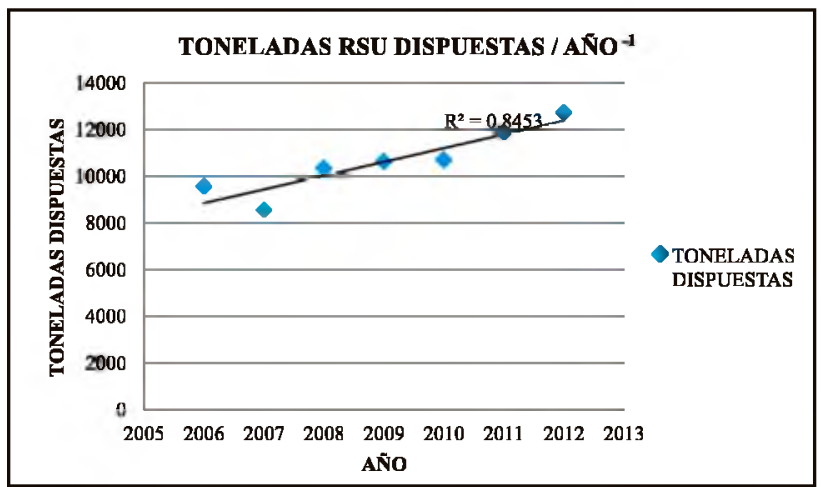

Figura 2. Toneladas dispuestas RSU Municipio de San Gil

La disposición final de los residuos se realiza en el relleno sanitario El Cucharo, ubicado en zona rural del municipio. Normalmente se dispone el $87 \%$ del total generado, la fracción restante corresponde a material reciclable que es aprovechado y comercializado por empresas legalmente constituidas hacía las principales ciudades del país. En la tabla 2 se observa las cantidades de GEI emitidas en el año 2006 y 2012 respectivamente debido a la descomposición de los RSU.

La producción per capital de San Gil es de 0,68 $\mathrm{kg} / \mathrm{hab} /$ día. Las proyecciones realizadas para el año 2012 en el PGIR están por debajo del valor real en 3.570 ton/año, demostrando que las autoridades y el municipio no están preparados en equipamiento e infraestructura para responder ante las consecuencias del crecimiento demográfico. 
En cuanto al componente de energía eléctrica, en la última década, ha aumentado hasta un $18 \%$ el consumo y un $4 \%$ el número de suscriptores. El 2002 el servicio fue a 14.329 suscriptores y el consumo promedio de hogar era de $100 \mathrm{kWh} / \mathrm{mes}$, para el año 2012 el servicio fue prestado a 14.927 suscriptores que corresponde al $99 \%$ de la población urbana y al $95 \%$ de la población rural y el consumo promedio por hogar alcanzó los $122 \mathrm{kWh} / \mathrm{mes}$ (ESSA, 2013). La energía es suministrada y distribuida por la ESSA a los suscriptores los cuales corresponden a hogares, principalmente; para las industrias locales como Cementos Argos, Protabaco y Cohilados del Fonce S.A la energía eléctrica es suministrada por ISAGEM, cuya red difiere a la del municipio. San Gil consume el $10 \%$ de la energía que se genera en la subestación de la localidad, pero en épocas decembrinas la demanda alcanza hasta el $40 \%$ del total generada; la demás cantidad es distribuida en los municipios aledaños.

Del componente aire se analizaron parámetros como material particulado y los resultados permiten concluir que están dentro de los rangos moderados (Resolución 601 de 2006, Resolución 610 de 2010); también se han realizado monitoreos para determinar la contaminación auditiva, incluso en día sin carro los resultados sobrepasan los límites permisibles. En la tabla 1 se relacionan los resultados del análisis realizado por la CAS en el año 2012.

Tabla 1. Informe Técnico de Medición de Ruido, CAS

\begin{tabular}{|c|c|c|c|c|c|}
\hline \multicolumn{6}{|c|}{ MEDICIÓN DE RUIDO MUNICIPIO DE SAN GIL } \\
\hline FECHA & RESL & DO & $\begin{array}{c}\text { NIVEL } \\
\text { PERMITIDO } \\
\text { (dB) }\end{array}$ & $\begin{array}{l}\text { HORA DE } \\
\text { INICIO }\end{array}$ & $\begin{array}{c}\text { HORA DE } \\
\text { TERMINACIÓN }\end{array}$ \\
\hline \multicolumn{6}{|c|}{ SEMANA DEL 30 DE JULIO AL 03 DE AGOSTO DE 2012} \\
\hline Agos-01 y 02 de 2012 & carrera 11 con calle 15 & 71,6 & 70 & $10: 44: 59$ & $10: 59: 31$ \\
\hline Agos-01 y 02 de 2012 & Esquina Iglesia San francisco & 74,9 & 70 & $11: 06: 21$ & $11: 21: 21$ \\
\hline Agos-01 y 02 de 2012 & Carrera 10 calle 13 & 68,8 & 65 & $11: 31: 45$ & $11: 46: 45$ \\
\hline Agos-01 y 02 de 2012 & Avenida Santander Entrada San Martin & 74 & 70 & $12: 07: 12$ & $12: 22: 12$ \\
\hline \multirow{3}{*}{$\begin{array}{l}\text { Agos- } 03 \text { de } 2012 \\
\text { (Día sin carro) }\end{array}$} & Carrera 11 con calle 10 & 74,3 & 65 & $10: 38: 19$ & $10: 53: 19$ \\
\hline & & & & & \\
\hline & \multicolumn{5}{|c|}{ SEMANA DEL O5 AL 10 DE AGOSTO DE 2012} \\
\hline Agos-08 y 09 de 2012 & carrera 11 con calle 15 & 73,7 & 65 & 10:05:22 & $10: 20: 22$ \\
\hline Agos-08 y 09 de 2012 & Esquina Iglesia San francisco & 75,1 & 70 & $10: 32: 48$ & $10: 47: 48$ \\
\hline Agos-08 y 09 de 2012 & Carrera 10 calle 13 & 70,2 & 65 & $11: 02: 12$ & $11: 17: 12$ \\
\hline Agos-08 y 09 de 2012 & Avenida Santander Entrada San Martin & 75,3 & 70 & $11: 42: 39$ & $11: 57: 39$ \\
\hline Agos-09 y 10 de 2012 & Carrera 11 con calle 10 & 75,6 & 65 & $10: 09: 25$ & $10: 24: 25$ \\
\hline
\end{tabular}

Sin embargo se desconoce el aporte en GEI el tránsito de vehículos particulares y de transporte público aporta a esta variable ambiental. No obstante existen otras actividades que generan afectación al componente aire con emisiones, sobresale la generación de los RSU, las actividades ganaderas y agrícolas, los procesos industriales, el uso de combustibles fósiles especialmente en fuentes móviles, el uso de aparatos electrónicos, entre otros.

Utilizando los factores de cálculo de emisiones del IPCC y la UPME se determinaron las generadas por los RSU y la demanda de energía eléctrica en los hogares del municipio en la última década, la tabla 2 muestra el aporte de estas fuentes a la generación de GEI. 
Tabla 2. Emisión de Ton/año GEI RSU y Electricidad

\begin{tabular}{|c|c|c|c|}
\hline \multicolumn{4}{|c|}{ RESIDUOS SÓLIDOS URBANOS (RSU) } \\
\hline AÑo & GEI Emitido & $\begin{array}{l}\text { FACTOR DE CONVERSIÓN } \\
\text { IPCC-UPME 2010- Kg/Tj }\end{array}$ & Ton.año ${ }^{-1}$ GEI Emitidas \\
\hline \multirow[t]{4}{*}{2006} & $\mathrm{CO}$ & 5000 & 512,9 \\
\hline & $\mathrm{CH} 4$ & 300 & 30,774 \\
\hline & $\mathrm{N}_{2} \mathrm{O}$ & 1 & 0.103 \\
\hline & TOTALAÑo & & $\mathbf{5 4 3 . 7 7 7}$ \\
\hline \multirow[t]{4}{*}{2012} & $\mathrm{CO}$ & 5000 & 728,56 \\
\hline & $\mathrm{CH} 4$ & 300 & 43,713 \\
\hline & $\mathrm{N}_{2} \mathrm{O}$ & 1 & 0,146 \\
\hline & TOTALAÑo & & 772.419 \\
\hline \multicolumn{4}{|c|}{ ELECTRICIDAD } \\
\hline AÑo & GEI Emitido & $\begin{array}{l}\text { FACTOR DE CONVERSIÓN } \\
\text { IPCC-UPME 2008- } 2010 \mathrm{Kg} / \mathrm{kWh}\end{array}$ & Ton.año-1 $\mathrm{CO}_{2}$ Emitidas \\
\hline 2002 & $\mathrm{CO}_{2}$ & 0,2717 & 4671,82 \\
\hline 2012 & $\mathrm{CO}_{2}$ & 0,2849 & 6123,891 \\
\hline
\end{tabular}

Con la identificación de las variables afectadas por el crecimiento demográfico, se evaluaron sus impactos utilizando la matriz de Leopold. La variable ambiental con mayor afectación es el agua, puesto que todas las acciones desarrolladas por los sangileños repercuten negativamente. Sin embargo el componente aire, debido a la generación de GEI y el cambio del uso del suelo se muestran significativamente afectados. En consecuencia se debe a las actividades agrícolas - pecuarias y las actividades domésticas urbanas la alteración de las variables ambientales, en la tabla 3 se muestra la matriz con la evaluación de impactos.

Tabla 3. Matriz de Leopold para evaluación de impactos ambientales.

\begin{tabular}{|c|c|c|c|c|c|c|c|c|c|c|}
\hline \multirow{2}{*}{$\begin{array}{l}\text { 푬 } \\
\text { 응 } \\
\text { 물 } \\
\text { 똥 }\end{array}$} & \multirow{2}{*}{\multicolumn{2}{|c|}{$\begin{array}{l}\text { 1. Las columnas indican las acciones del } \\
\text { hombre susceptibles de alterar las variables } \\
\text { ambientales. } \\
\text { 2. Las filas son las variables ambientales a } \\
\text { evaluar. } \\
\text { 3.Las ponderaciones se dan en magnitud } \\
\text { Vs. Importancia }\end{array}$}} & \multicolumn{7}{|c|}{ ACCIONES QUE PUEDEN CAUSAR IMPACTOS AMBIENTALES } & \multirow{3}{*}{ 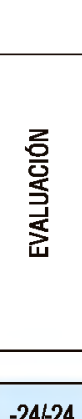 } \\
\hline & & & 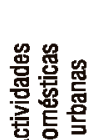 & 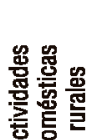 & 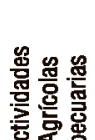 & 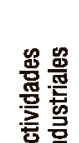 & 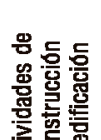 & 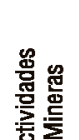 & 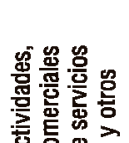 & \\
\hline \multirow{12}{*}{$\begin{array}{l}\text { 出崫 } \\
\text { 产 } \\
\text { 垔 }\end{array}$} & AGUA & Superficial & $-5 /-5$ & $-4 /-4$ & $-4 /-4$ & $-3 /-3$ & $-2 /-2$ & $-4 /-4$ & $-2 /-2$ & \\
\hline & \multirow{3}{*}{ AIRE } & Material Particulado & $-2 /-2$ & $-3 /-3$ & $-2 /-2$ & $-4 / 4$ & $-4 /-3$ & $-1 /-1$ & $-11-1$ & $-17 /-16$ \\
\hline & & GEI & $-5 /-5$ & $-3 /-2$ & $-4 /-4$ & $-5 /-5$ & $-1 /-1$ & $-1 / 1$ & $-11-1$ & $-20 /-19$ \\
\hline & & \begin{tabular}{|l|l|} 
Ruido \\
\end{tabular} & $-2 /-2$ & $0 / 0$ & $0 / 0$ & $-4 / 3$ & $-3 /-2$ & $0 / 0$ & $-41-5$ & $-13 k-12$ \\
\hline & \multirow{2}{*}{ SUELO } & Cambio de uso & $-3 /-3$ & $-1 /-1$ & $-4 /-4$ & $-1 /-1$ & $-4 /-3$ & $-3 /-3$ & $-11-1$ & $-17 /-16$ \\
\hline & & \begin{tabular}{|l} 
Explotación \\
\end{tabular} & $-3 /-3$ & $-1 /-1$ & $-4 /-4$ & $0 / 0$ & $-1 /-1$ & $-4 /-4$ & $0 / 0$ & $-13 /-13$ \\
\hline & \multirow{3}{*}{ FLORA } & Especies nativas & $0 / 0$ & $-2 /-2$ & $-3 /-2$ & $0 / 0$ & $-2 /-2$ & $0 / 0$ & $-1 /-1$ & $-8 / 7$ \\
\hline & & \begin{tabular}{|l|} 
Bosques \\
\end{tabular} & $0 / 0$ & $0 / 0$ & $-3 /-3$ & $0 / 0$ & $0 / 0$ & $0 / 0$ & $0 / 0$ & $-3 /-3$ \\
\hline & & Zonas delimitadas & $0 / 0$ & $0 / 0$ & $-2 /-2$ & $-1 /-1$ & $-1 /-1$ & $0 / 0$ & $0 / 0$ & $-4 /-4$ \\
\hline & \multirow{2}{*}{ FAUNA } & Especies nativas & $0 / 0$ & $-1 /-1$ & $-11-1$ & $0 / 0$ & $0 / 0$ & $0 / 0$ & $0 / 0$ & $-2 /-2$ \\
\hline & & \begin{tabular}{|l} 
Especies en vla de extinciôn \\
\end{tabular} & $0 / 0$ & $-1 /-1$ & $-11-1$ & $0 / 0$ & $0 / 0$ & $0 / 0$ & $0 / 0$ & $-2 /-2$ \\
\hline & ENERG|A & \begin{tabular}{|l} 
Consumo energético eléctrico \\
\end{tabular} & $-4 /-4$ & $-3 /-3$ & $0 / 0$ & $0 / 0$ & $0 / 0$ & $0 / 0$ & $0 / 0$ & $-71-7$ \\
\hline \multicolumn{3}{|c|}{ EVALUACIÓN } & $-24 /-24$ & $-19 /-19$ & $-28 /-28$ & $-18 /-18$ & $-18 /-15$ & $-13 /-13$ & $-10<-11$ & \\
\hline
\end{tabular}




\subsection{Lineamientos de gestión}

Para orientar el desarrollo municipal de acuerdo a las problemáticas analizadas como consecuencia del crecimiento demográfico, se propusieron lineamientos de gestión, los cuales comprenden:

$\checkmark$ Gestión y planeación conjunta de programas dirigidos al aprovisionamiento de bienes y servicios ambientales desligado del crecimiento demográfico.

$\checkmark$ Para atender la demanda de servicios de calidad mejoramiento de equipamiento e infraestructura a través de monitoreo y seguimiento constante de fuentes generadoras y abastecedoras bajo conceptos de sostenibilidad.

$\checkmark$ Implementación de conceptos tecnológicos que permita realizar aprovechamiento de materiales propios y generación de nuevos mercados propendiendo por la implementación de alternativas innovadoras y la disminución de emisiones.

$\checkmark \quad$ Identificación y delimitación de zonas especiales y de uso público en la municipalidad bajo conceptos legales, identificando especialmente la margen de crecimiento urbanístico.

\section{Conclusiones}

La variable ambiental más afectada por el incremento demográfico en el municipio de San Gil en los últimos 10 años es el agua, sin embargo variables como aire, $y$ suelo también muestran una significativa afectación.

De acuerdo a la evaluación de impactos, las actividades asociadas a la producción agrícola y acciones domésticas urbanas son las que más afectan las variables ambientales.

Al aumentar el crecimiento demográfico aumenta el número de consumidores y por ende la utilización de bienes y servicios ambientales para atender la demanda. En el municipio de San Gil, el aumento de la población en la última década incrementó la demanda de bienes y servicios ambientales en no menos del $30 \%$ y en igual proporción las emisiones de GEI.

El incremento demográfico dificulta el préstamo de servicios de calidad; entre más población haya concentrada en un espacio geográfico, mayor la dificultad de poner a disposición bienes y servicios bajo conceptos de calidad y sostenibilidad. El equipamiento e infraestructura diseñado a comienzos de la última década para la prestación de servicios, no contemplo el significativo aumento de la población en especial la flotante.

La generación de residuos sólidos y el uso de energía eléctrica en el municipio de San Gil provocó emisiones de GEI acumuladas por $25.175,605$ Ton/año en la última década.

\section{Referencias Bibliográficas}

1. Arias, E., 2010. Año Internacional de la biodiversidad. Retos y Oportunidades hacia 2020. Memorias 2010. Instituto de Investigación de Recursos Biológicos Alexander von Humboldt. Ediprint Ltda, Bogotá, 160 pp.

2. Bloom, H., 2001, Shakespeare. La invención de lo humano, Editorial Norma, Bogotá D.C.

3. Colombia,Alcaldía municipal de San Gil, oficina de cultura y turismo municipal, 2012.

4. Colombia, Alcaldía municipal de San Gil, oficina de planeación municipal, 2012.

5. Colombia, Alcaldía municipal de San Gil "Plan Básico de Ordenamiento Territorial", 2003.

6. Colombia, Alcaldía municipal de San Gil "Plan de Desarrollo 2012-2015", 2012.271 p.

7. Colombia, Corporación Autónoma Regional de Santander CAS, "Informe Técnico de Medición de Ruido" 20134 pág.

8. Colombia, Electrificadora de Santander ESSA, Oficina San gil, 2013.

9. Colombia, Fundación Universitaria de San Gil UNISANGIL, Gobernación de Santander "Plan Prospectivo Guanentá 2025" 201036p.

10. Georg, K, 2002. Factores determinantes de la Contaminación Ambiental y del Uso de los Recursos Naturales. Consultado 26 de mayo de 2012. $\mathrm{http}: / /$ www.redalyc.org/articulo.oa? $\mathrm{id}=81820111$.

11. Michael, A., 1999. citado por Manrique F. et.al,. 2007. Crecimiento Poblacional y Políticas Públicas. Consultado 22 de abril de 2012.

https://www.google.com.co/webhp?sourceid= chrome-instant\&ion $=1$ \&espv $=2 \&$ ie $=U T F-8 \# q=$ crecimiento + poblacional $+y+$ politicas + publicas

12. Ponce, E., 2010. Año Internacional de la biodiversidad. Retos y Oportunidades hacia 2020. Memorias 2010. Instituto de Investigación de Recursos Biológicos Alexander von Humboldt. Ediprint Ltda, Bogotá, 27-28

13. Weltbank, 1992. Citado por Georg K., 2002. Factores determinantes de la Contaminación Ambiental y del Uso de los Recursos Naturales. Consultado 26 de mayo de 2012.

http://www.redalyc.org/articulo.oa?id=81820111. 